NASA Technical Memorandum 103630

ICOMP-90-24

\title{
Mappings and Accuracy for Chebyshev Pseudo-spectral Approximations
}

Alvin Bayliss and Eli Turkel

Institute for Computational Mechanics in Propulsion Lewis Research Center

Cleveland, Ohio

December 1990

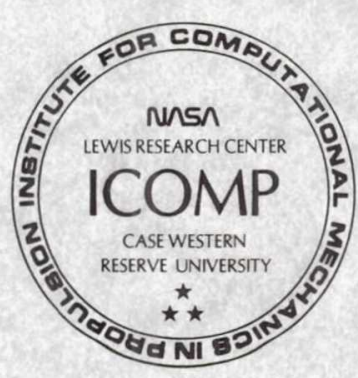




\title{
Mappings and Accuracy for Chebyshev Pseudo-spectral Approximations*
}

\author{
Alvin Bayliss $\dagger$ and Eli Turkel $\ddagger$ \\ Institute for Computational Mechanics in Propulsion \\ Lewis Research Center \\ Cleveland, Ohio 44135
}

\begin{abstract}
The effect of mappings on the approximation, by Chebyshev collocation, of functions which exhibit localized regions of rapid variation is studied. A general strategy is introduced whereby mappings are adaptively constructed which map specified classes of rapidly varying functions into low order polynomials which can be accurately approximated by Chebyshev polynomial expansions. A particular family of mappings constructed in this way is tested on a variety of rapidly varying functions similar to those occurring in approximations. It is shown that the mapped function can be approximated much more accurately by Chebyshev polynomial approximations than in physical space or where mappings constructed from other strategies are employed.
\end{abstract}

\section{Introduction.}

One of the major difficulties in the application of Chebyshev pseudo-spectral methods, or other spectral methods, to the solution of partial differential equations, is in the approximation of functions which exhibit localized regions of rapid variation.

*Work funded under Space Act Agreement C-99066-G.

tNorthwestern University, Evanston, Illinois.

$\ddagger$ Tel-Aviv University, Tel-Aviv, Israel. Work also partially funded by National Science Foundation grants ASC 87-19573 and DMS 87-01543. 
The approximation of such functions by polynomial expansions generally results in global oscillations unless a large number of terms are used in the polynomial approximation. These oscillations often lead to instabilities and inaccuracies when spectral methods are applied to the solution of partial differential equations $[8,11]$. One possible remedy is to introduce a mapping to a new coordinate system so that the mapped function can be more accurately approximated by low order polynomial expansions.

There is a great deal of computational evidence that appropriately chosen mappings can significantly enhance the accuracy of pseudo-spectral applications. In [16] mappings were shown to enhance the accuracy of approximations to shock like (hyperbolic tangent) functions. In $[1,3,5,9]$ mappings were introduced dynamically via an adaptive procedure in which some error measure of the solution was minimized. Mappings have also been used to approximate boundary layer flows in Navier-Stokes calculations, for example [8].

There are two issues that must be addressed in the systematic application of mappings to enhance the accuracy of Chebyshev pseudo-spectral methods. These are

- The construction of appropriate family of mappings

- Criteria to choose a particular mapping from this family according to the behavior of the solution to be approximated.

The second issue has been addressed by employing adaptive procedures in order to determine appropriate mappings $[1,3,5,9]$. In these procedures, a family of mappings is introduced depending on a small number of free parameters. In addition functionals are derived which are used to monitor the pseudo-spectral error. The appropriate mapping function is then chosen so that when the problem is transformed by the mapping the functional is minimized.

The choice of an appropriate functional to monitor the pseudo-spectral error is an important component of the adaptive procedure. In $[5,9]$ a Sobolev type norm of the solution was chosen as the functional monitoring the pseudo-spectral error. In [3] another functional, derived directly from the Chebyshev expansion of an arbitrary function, was used in an adaptive procedure and shown to be more effective than the Sobolev norm in measuring the errors of the pseudo-spectral approximation.

Another equally important component of the adaptive procedure is the choice of the family of mappings to be utilized as coordinate transformations. Usually, the family of coordinate transformations to be incorporated in the solution method is prescribed in advance and justified by, at most, heuristic reasoning. There have been very few detailed studies of the effects of different families of mappings on the accuracy of pseudo-spectral approximations of various different types of functions. In [13] a family of mappings was derived which had the effect of giving a more uniform distribution to the collocation points, i.e. in an appropriate (but singular) limit the mapped Chebyshev collocation points were uniformly distributed. Numerical examples demonstrated that this mapping function gave enhanced accuracy over 
the unmapped Chebyshev pseudo-spectral method for functions which did not have rapid variations. However the effect of the mapping on functions exhibiting rapid variation was not considered. A similar type of mapping was presented in [1]. In [3] a family of mappings was introduced which attempted to map certain interior regions to the boundaries.

In this paper we derive a family of mappings which are well suited to the approximation of functions with localized regions of rapid variations. The family of mappings depend on two parameters which are related to the size of the gradient over the narrow interval and the location of the region of rapid variation respectively. The effect of this mapping on the accuracy of a Chebyshev approximation is analyzed for a variety of different functions and compared to the families of mappings in $[3,13]$ and to the case where no mapping is employed.

\section{Pseudo-Spectral Approximation and Mappings}

We first describe the derivation of the Chebyshev pseudo-spectral approximation. This discussion is brief, a more detailed description may be found in $[8,11]$. Let $f(x)$ be a defined in the interval $I,-1 \leq x \leq 1$. The Gauss-Lobatto points are defined as

$$
x_{j}=\cos \left(\frac{j \pi}{N}\right), \quad 0 \leq j \leq N .
$$

$P_{n}$ the polynomial of $n^{\text {th }}$ degree which interpolates $f$ at the points $x_{j}$ is the Chebyshev interpolant, or pseudo-spectral, approximation to $f$ and can be obtained by using the Gauss-Lobatto quadrature rule to evaluate the expansion coefficients. It is known that the maximum norm error in approximating $f$ by the pseudo-spectral approximation differs by at most a factor $O(\log N)$ from the maximum norm error in approximating $f$ by the minimax polynomial [14]. A similar result is true for the Galerkin polynomial approximation to $f$. We anticipate that the results presented here are equally valid for Galerkin approximations.

However, in general a polynomial expansion is not appropriate to approximate functions with large gradients, e.g. functions exhibiting spikes, localized oscillations, or near discontinuities. Furthermore, the accuracy of Chebyshev approximations tends to be sensitive to the location of regions with large gradients. There is computational evidence that Chebyshev approximations are more accurate for functions which vary rapidly near the boundaries as opposed to functions which vary away from the boundaries, for example [16]. It was shown in [16] that this is true both for Chebyshev methods, where the collocation points cluster near the boundaries, and also for collocation based on evenly spaced points. It appears from the results in [16] that this is a property of polynomial approximations and is not necessarily related to the clustering of the collocation points.

We denote the family of mappings by,

$$
x=q(s, \alpha),
$$

where $x$ represents the physical coordinate, $-1 \leq s \leq 1$ is the transformed coordinate, and $\alpha$ denotes one or possibly more free parameters. The Chebyshev 
interpolant can then be used to approximate the transformed function $f(q(s, \alpha))$. The effect of the mapping can be regarded as transforming the function to be approximated to $f(q(s, \alpha))$ from $f(x)$. If the mapping is properly chosen $f(q(s, \alpha))$ will vary more gradually and so be more readily approximated by a polynomial. A different viewpoint is that in the original coordinate, $x$, we are changing the basis functions from polynomials to a new set of basis functions that can better represent functions that strongly vary in localized regions.

The mappings can improve the accuracy of the pseudo-spectral approximation in three general ways which can be employed as strategies in designing families of mappings. The transformed function can be less rapidly varying in the new coordinate system so it is better approximated by a polynomial. Second the region of rapid variation can be mapped to the boundaries. Third the mapping can expand the region near the boundaries thus tending to provide a more uniform distribution of collocation points. This latter approach was proposed in [13]. The distinction between these three potential strategies is not sharp, for example a mapping in which a spike at $x=0$ is transformed to a less rapidly varying function must of necessity map regions in the interior closer to the boundaries. However in this paper we will compare three families of mappings which were constructed based on these three potential strategies for several functions similar to those which occur in applications. Our results will demonstrate that in most instances mappings based on transforming the function into one which varies more gradually, tends to yield more accurate approximations.

When the Chebyshev pseudo-spectral method is used to solve partial differential equations there are many sources of errors and it is difficult to differentiate the source of the errors. In this paper we concentrate on the interpolation problem and evaluate the effectiveness of different mappings in reducing the maximum norm error in the pseudo-spectral approximation. In such a program one could simply start with a function and find the mapping parameters which yield the smallest error in some norm. However in applications the exact solution is not known and some adaptive procedure is necessary in order to select appropriate mapping parameters. In this paper we will determine mapping parameters by minimizing a functional related to the spectral interpolation error. This functional, derived in [3], is

$$
I_{2}(g)=\left(\int_{-1}^{1}\left(L^{2} g\right)^{2} / w(s) d s\right)^{\frac{1}{2}},
$$

where

$$
w(s)=\sqrt{1-s^{2}}, \quad L=w(s) \frac{d}{d s} .
$$

It can be shown that (3) is equivalent to the standard Sobolev norm under the mapping $x=\cos (\theta)$. Furthermore, this functional gives an upper bound on the maximum norm of the error [3].

Our approach will be to compute (3) using the transformed function $f(q(s, \alpha))$. We will do this by forming the Chebyshev interpolant to $f(q(s, \alpha))$ using a fixed value of $N$ and then, using the Gauss-Lobatto quadrature formula, evaluating (3). The 
result will be a function only of the parameter vector $\alpha$ which we will denote by $I_{2}(\alpha)$. We choose $\alpha$ so as to minimize $I_{2}$. In practice this minimum is found by computing $I_{2}(\alpha)$ over a range of values of $\alpha$ and then choosing that parameter for which a minimum occurs. In most cases we will take the global minimum over the range of values of $\alpha$. In certain circumstances global minima occur for parameters which give a highly inaccurate approximation. This is due to inaccuracies in computing the quadrature in (3) when the transformed function $f(q(s, \alpha))$ is poorly approximated for some parameters $\alpha$. In this case we take local minima for which the Chebyshev interpolant is a good approximation to the given function $f$.

This approach is a practical and effective method to determine mappings which enhance the accuracy of the pseudo-spectral approximation [3]. It has been successfully utilized in the computation of problems in combustion $[3,5,9]$ and has also been used for problems in wave propagation in [1]. Furthermore in all cases presented below the errors that were obtained from mappings for which $I_{2}(\alpha)$ was minimized were very close to the minimum errors in the maximum norm over the range of parameters investigated.

\section{Families of Mappings}

We first introduce a family of mappings which have the property of transforming functions of a certain class to polynomials. Although the functions occuring in applications will not be exactly of the specified class, they would be expected to be sufficiently similar so that the Chebyshev approximation will require only low degree polynomials to give good accuracy.

We begin with a specified class of functions of the form $s=h\left(x, \alpha_{1}, \alpha_{2}\right)$. We suppose for certain parameter values this function exhibits rapid variations. For example suppose that for large values of $\alpha_{1}$, this function exhibits shock-like behavior with the rapid variations occuring near the point $x=\alpha_{2}$ and nearly constant behavior away from this point. If the function $h\left(x, \alpha_{1}, \alpha_{2}\right)$ is univalent then upon an appropriate rescaling this function can be assumed to map the interval $-1 \leq x \leq 1$ univalently onto itself. The inverse function $x=h^{-1}\left(s, \alpha_{1}, \alpha_{2}\right)$ then describes a family of mappings such that under this mapping the function $h$ becomes linear and can therefore be approximated by a low order expansion of Chebyshev polynomials. In applications the rapidly varying solutions will not be exactly of the form of the given function $h\left(x, \alpha_{1}, \alpha_{2}\right)$, however the image of these functions under the mapping given by $h^{-1}$ is likely to have a gradual variation and so can be approximated by a low order polynomial expansion provided the parameters $\alpha_{1}$ and $\alpha_{2}$ are properly chosen. This will be justified by the examples presented below.

The mapping $x=h^{-1}\left(s, \alpha_{1}, \alpha_{2}\right)$ can be expected to enhance the approximation not only rapidly varying functions similar to $h\left(x, \alpha_{1}, \alpha_{2}\right)$ but also rapidly varying functions which behave like derivatives of $h\left(x, \alpha_{1}, \alpha_{2}\right)$. In order to see why this is so let $g=h^{\prime}\left(x, \alpha_{1}, \alpha_{2}\right)$ where ' denotes the derivative with respect to $x$. If $h\left(x, \alpha_{1}, \alpha_{2}\right)$ behaves as a step function then $g\left(x, \alpha_{1}, \alpha_{2}\right)$ will behave as a spike centered at $x=\alpha_{2}$. Suppose that this point is located away from the boundaries. Using the chain rule 
we obtain an expression for $g\left(x, \alpha_{1}, \alpha_{2}\right)$ under the mapping $s=h\left(x, \alpha_{1}, \alpha_{2}\right)$,

$$
g\left(h^{-1}\left(s, \alpha_{1}, \alpha_{2}\right), \alpha_{1}, \alpha_{2}\right)=\left(\partial h^{-1}\left(s, \alpha_{1}, \alpha_{2}\right) / \partial s\right)^{-1} .
$$

If the function $h\left(x, \alpha_{1}, \alpha_{2}\right)$ behaves as a step function changing rapidly near the interior point $x=\alpha_{2}$, then clearly $\partial h^{-1}\left(s, \alpha_{1}, \alpha_{2}\right) / \partial s$ will change most rapidly near the boundaries in $s$. Therefore we expect the mapping to enhance the accuracy of the approximation for $g$. Similar arguments hold for the approximation of functions similar to higher derivatives of $h\left(x, \alpha_{1}, \alpha_{2}\right)$.

In this paper we consider the following function $h\left(x, \alpha_{1}, \alpha_{2}\right)$

$$
s=s_{0}+\tan ^{-1}\left(\alpha_{1}\left(x-\alpha_{2}\right)\right) / \lambda .
$$

For large values of $\alpha_{1}$ this function is nearly discontinuous with a region of rapid variation occuring near $x=\alpha_{2}$. The parameters $s_{0}$ and $\lambda$ are determined so that (5) maps the interval $I$ onto itself. The values of $s_{0}$ and $\lambda$, determined in this way are

$$
\begin{gathered}
s_{0}=\frac{\kappa-1}{\kappa+1}, \kappa=\tan ^{-1}\left(\alpha_{1}\left(1+\alpha_{2}\right)\right) / \tan ^{-1}\left(\alpha_{1}\left(1-\alpha_{2}\right)\right), \\
\lambda=\tan ^{-1}\left(\alpha_{1}\left(1-\alpha_{2}\right)\right) /\left(1-s_{0}\right) .
\end{gathered}
$$

With these choices of $\lambda$ and $s_{0}$ the inverse of (5),

$$
x=\alpha_{2}+\tan \left(\left(s-s_{0}\right) \lambda\right) / \alpha_{1},
$$

describes a two-parameter family of mappings of $I$ into itself which is suitable for the accurate resolution of functions with localized regions of rapid variation. Here $\alpha_{1}$ is related to the degree of change of the function and $\alpha_{2}$ is related to the location of the region of rapid variation. In applications these parameters would would be obtained either from prior knowledge of the solution or from minimizing a functional such as (3) which measures the error in the approximation. We note that (6) is explicitly invertible.

For small $\alpha_{1}, s$ is approximately equal to $x$ and (6) is approximately the identity map. The use of the mapping (6) should not therefore be expected to degrade the accuracy in approximating slowly varying functions which can be well approximated by a Chebyshev approximation without any mapping.

We point out that the an alternative to the inverse tangent function is the hyperbolic tangent function with an appropriate scaling. Mappings constructed using the hyperbolic tangent are related to stationary shock-like solutions of the Burgers equation [6].

An alternative family of mappings has been proposed in [13]. This family of mappings is

$$
x=\arcsin \left(\alpha_{1} s\right) / \arcsin \left(\alpha_{1}\right)
$$

and $0 \leq \alpha_{1} \leq 1$. The effect of this mapping is to expand the boundary regions and compress the interior regions. As $\alpha_{1} \rightarrow 1$ the image of the Gauss-Lobatto 
points become uniformly spaced however the mapping becomes singular. As $\alpha_{1} \rightarrow 0$ (7) approaches the identity mapping. The analysis and examples presented in [13] demonstrate that the use of (7) with $\alpha_{1}$ near 1 can significantly improve the accuracy of pseudo-spectral approximations for a range of functions. This was attributed to fact that the collocation points become nearly uniformly spaced as $\alpha_{1} \rightarrow 1$. The major effect of this mapping is to obtain a more uniform distribution of the collocation points and as such the mapping depends on only one parameter (however a two-parameter extension was proposed in [13]). A related mapping is presented in [1] which permits a concentration of collocation points in the interior which exceeds that at the boundary, i.e. it goes beyond obtaining a uniform distribution of the collocation points.

A third family of mappings that we will consider is

$$
s=4 / \pi \tan ^{-1}\left[\alpha_{1} \tan (\pi / 4)\left(x^{\prime}-1\right)\right]+1
$$

with

$$
x^{\prime}=\left(\alpha_{2}-x\right) /\left(\alpha_{2} x-1\right) .
$$

The use of this mapping is described in $[3,5]$. The effect of the mapping is to map an arbitrary point, $\alpha_{2}$, to the origin and then expand one of the boundaries at the expense of the other by varying $\alpha_{1}$. This mapping is presented in [5] where it was used with $\alpha_{2}=0$ to compute functions with rapid variations near the boundary and in [3] to compute functions with rapid variations in the interior.

\section{Mappings and Accuracy}

In this section we compare the effectiveness of the families of mappings described by (6), (7), and (8) in enhancing the accuracy of pseudo-spectral approximations. Our approach is to construct the pseudo-spectral polynomial for the transformed function and then to measure the maximum norm of the error. The maximum norm of the error is computed by comparing the approximating polynomial and the given function over a large grid of points. We also compute the discrete $L_{2}$ (unweighted) norm of the error in the coordinate system selected. We note that the $L_{2}$ norm of the error depends on the coordinate system and may not adequately measure the relevant errors in applications.

We first consider approximations to the function

$$
y=\tanh \left(\sigma\left(x-x_{0}\right)\right) .
$$

with $\sigma=40$. In our computations we compute over a grid of values of $\alpha_{1}$ and $\alpha_{2}$ and select those parameter values for which the functional $I_{2}$ is minimized. For the case of (6) the global minimum always occurs when $\alpha_{2}=x_{0}$, however, we indicate the sensitivity of the approximation to the value of $\alpha_{2}$. The approximating polynomial is computed using $N=121$. The error is computed using the Gauss-Lobatto points with $N=351$. Both the maximum norm and the discrete $L_{2}$ norm of the error are computed. The results are presented in Table 1 . An entry of $U$ in the first column denotes that no mapping was used. 


\begin{tabular}{||c|c|c|c|c|c||}
\hline Mapping & $\alpha_{1}$ & $\alpha_{2}$ & $I_{2}$ & Max Error & $L_{2}$ Error \\
\hline 6 & 51.30 & 0. & 2.40 & $1.71 \mathrm{e}-14$ & $1.10 \mathrm{e}-14$ \\
\hline 6 & 6.06 & .2 & 80.57 & $8.37 \mathrm{e}-05$ & $2.18 \mathrm{e}-05$ \\
\hline 7 & .99999 & NA & 132.54 & $1.51 \mathrm{e}-03$ & $3.48 \mathrm{e}-04$ \\
\hline 7 & .9 & NA & 181.24 & $6.72 \mathrm{e}-03$ & $1.42 \mathrm{e}-03$ \\
\hline 8 & 9.92 & -.887 & 189.12 & $7.92 \mathrm{e}-03$ & $1.70 \mathrm{e}-03$ \\
\hline U & NA & NA & 227.14 & $2.05 \mathrm{e}-02$ & $4.02 \mathrm{e}-03$ \\
\hline
\end{tabular}

Table 1: $y=\tanh \left(\sigma\left(x-x_{0}\right)\right)$

The data in this table illustrates the effectiveness of the mapping (6) in enhancing the approximation of the shock-like profile (9). A graphical illustration of this is shown in Figures 1a and 1b. In Figure 1a we plot (9) against the original independent variable $x$. In Figure 1b we plot the transformed function against the variable $s$ using $\alpha_{1}=51.30$ and $\alpha_{2}=0$. The figures clearly illustrate the more gradual variation of the transformed function.

We note that the mapping (7) allows for a more accurate approximation than if no mapping at all is employed and also permits a considerably more accurate approximation than the use of (8). The best accuracy (and the minimum value of (3)) occurs as $\alpha_{1} \rightarrow 1$ so that the collocation points are uniformly spaced but the mapping is singular. Values of $\alpha_{1}$ close to 1 do not affect our approximation program, however when mappings are applied to the solution of partial differential equations, Jacobians are introduced into the equations. The singularity in the mapping (7) which occurs at $\alpha_{1}=1$ may therefore effect the solution of partial differential equations when $\alpha_{1}$ is sufficiently close to 1 . In the solution of partial differential equations the degree of 'near singularity' that can be allowed in the mapping without affecting the numerical approximation will be problem dependent, depending on other parameters of the solution process not evaluated here. There is a degradation in accuracy as $\alpha_{1}$ is reduced from the singular value 1 . We have presented results for $\alpha_{1}=.9$ in order to illustrate the behavior of the approximation as $\alpha_{1}$ is varied.

Finally we note that (6) is robust in the sense that even if $\alpha_{2}$ is not at the point of maximum variation it still yields a significantly improved approximation. This is important for several reasons.

- The minimization might be inexact.

- There may be several regions of rapid variation clustered together.

- Different dependent variables might exhibit rapid spatial variation at different points.

- In many two dimensional problems there is often a principal direction in which the spike (or rapid variation) occurs, however the location of this variation 


\begin{tabular}{||c|c|c|c|c|c||}
\hline Mapping & $\alpha_{1}$ & $\alpha_{2}$ & $I_{2}$ & Max Error & $L_{2}$ Error \\
\hline 6 & 51.30 & 0. & 2.40 & $2.20 \mathrm{e}-11$ & $1.70 \mathrm{e}-11$ \\
\hline 6 & 6.06 & .2 & 80.95 & $1.96 \mathrm{e}-03$ & $6.68 \mathrm{e}-04$ \\
\hline 7 & .99999 & NA & 119.22 & $1.60 \mathrm{e}-02$ & $4.08 \mathrm{e}-03$ \\
\hline 8 & 9.92 & -.887 & 137.60 & $5.11 \mathrm{e}-02$ & $1.12 \mathrm{e}-02$ \\
\hline U & NA & NA & 130.77 & $8.68 \mathrm{e}-02$ & $1.92 \mathrm{e}-02$ \\
\hline
\end{tabular}

Table 2: $y=\tanh \left(\sigma\left(x-x_{0}\right)\right)$, using $N=81$

may depend on some transverse coordinate. It may be more efficient to employ a one-dimensional mapping to resolve such a solution rather than a twodimensional mapping which depends on the transverse coordinate.

The behavior described in the last point above is typical of partial differential applications, for example in wave propagation if a pulse propagates through a dispersive medium, in fluid dynamics if curved shocks form and in combustion where cells can form along flame fronts. Thus we consider the robustness of the mapping (6) with respect to the location parameter $\alpha_{2}$ to be an important point.

We next illustrate the convergence properties for the mapped function by considering the same results using $N=81$. The results are presented in Table 2. In this case we have used the same mapping parameters as were obtained using 121 collocation points. The results show that there is a much more rapid convergence when the mapping (6) is used. This is probably due to infinite order convergence of the spectral method which in practice means a very high order of convergence when the function varies gradually. We note that there are definite numerical errors in the approximation of the function $I_{2}$ when the mapping (6) is not used. In practice this could lead to poor parameter values being produced by the minimization procedure.

The results described for (9) are not necessarily surprising as the family of mappings (6) is constructed so that the image of a near step function is linear. We next consider the function

$$
y=\exp \left(\sigma^{2}\left(x-x_{0}\right)^{2} / 2\right) .
$$

This function also exhibits a rapid variation near $x=x_{0}$ however the behavior is that of an isolated spike rather than that of a near step as in (9). We consider the values $\sigma=50$ and $x_{0}=0$. The results are presented in Table 3 .

We can infer from the results presented in Table 3 that the mapping (6) is effective in enhancing the resolution of spike-like functions. We also illustrate this graphically in Figures $2 \mathrm{a}$ and $2 \mathrm{~b}$ where we plot the function (10) in the original coordinate, $x$, (Figure 2a) and the transformed function in the mapped coordinate, $s$, (Figure 2b). We note that the mapped function still resembles a Gaussian, however there is a much more gradual variation.

The results also illustrate that the use of the mapping (6) provides good accuracy as the parameter $\alpha_{2}$ is moved away from the location of the spike. The results 


\begin{tabular}{||c|c|c|c|c|c||}
\hline Mapping & $\alpha_{1}$ & $\alpha_{2}$ & $I_{2}$ & Max Error & $L_{2}$ Error \\
\hline 6 & 37.48 & 0. & 18.26 & $1.42 \mathrm{e}-09$ & $8.30 \mathrm{e}-10$ \\
\hline 6 & 10.86 & .1 & 106.67 & $3.37 \mathrm{e}-06$ & $1.48 \mathrm{e}-06$ \\
\hline 6 & 5.744 & .2 & 245.51 & $1.59 \mathrm{e}-03$ & $5.51 \mathrm{e}-04$ \\
\hline 6 & 3.506 & .3 & 386.86 & $2.20 \mathrm{e}-02$ & $6.08 \mathrm{e}-03$ \\
\hline 7 & .99999 & NA & 285.20 & $1.20 \mathrm{e}-02$ & $6.06 \mathrm{e}-03$ \\
\hline 7 & .9 & NA & 557.02 & $4.90 \mathrm{e}-02$ & $1.96 \mathrm{e}-02$ \\
\hline 8 & 73.92 & -.983 & 547.04 & $4.57 \mathrm{e}-02$ & $1.85 \mathrm{e}-02$ \\
\hline U & NA & NA & 699.84 & $1.14 \mathrm{e}-01$ & $3.95 \mathrm{e}-02$ \\
\hline
\end{tabular}

Table 3: $y=\exp \left(\sigma^{2}\left(x-x_{0}\right)^{2} / 2\right), x_{0}=0$

suggest that the mapping (6) can allow high resolution of multiple spikes even when the parameter $\alpha_{2}$ deviates significantly from the location of each individual spike. Of course if the spikes are widely separated or if $\alpha_{2}$ is far from the center of the spike the effectiveness of (6) degrades. We have found that the accuracy obtained from using (6) does not strongly depend on the value of $x_{0}$ provided $\alpha_{2}$ is chosen to be near $x_{0}$. Similar results hold for functions exhibiting a step function type of behavior as in (9). We also note that the mapping (7) provides considerably more accuracy than (8) indicating that improvements over the original Chebyshev method when the boundary clustering of the collocation points is reduced.

The results in Table 3 indicate that the functional (3) has a rather sharp minimum when $\alpha_{2}$ is at the location of the spike. In our calculations we have found the value of $\alpha_{2}$ obtained from minimizing (3) to be an excellent indicator of the location of the spike (or most rapid variation). This suggests that this procedure could be used as a shock locator for non-oscillatory spectral methods and filtering methods $[7,10]$, at least when only one shock is present or multiple shocks are closely spaced.

The effect of the location of the spike does considerably effect the behavior obtained from using (7) and (8). We illustrate this in our next example where we consider a spike near the boundary by setting $x_{0}=.9$. The results are presented in Table 4.

The mapping (6) still gives very good accuracy even when $\alpha_{2}$ is not close to $x_{0}$. The best approximation using (7) now occurs when $\alpha_{1} \rightarrow 0$ where the mapping approaches the identity. In Table 4 we indicate the errors found for $\alpha_{1}=.0001$, the limits of our search region together with the errors for larger values of $\alpha_{1}$ which lead to approximately uniformly spaced points. For functions which have significant variations near the boundaries having the collocation points more uniformly spaced leads to a degradation in accuracy. We find that the behavior of the mapping (7) depends very abruptly on the location of the spike. For values of $x_{0}$ just below .8 we find an abrupt transition where the value of $\alpha_{1}$ yielding maximum accuracy switches from $\alpha_{1}$ near 1 to $\alpha_{1}$ near 0 . In this narrow overlap region the results obtained from 


\begin{tabular}{||c|c|c|c|c|c||}
\hline Mapping & $\alpha_{1}$ & $\alpha_{2}$ & $I_{2}$ & Max Error & $L_{2}$ Error \\
\hline 6 & 35.25 & .9 & 15.05 & $3.29 \mathrm{e}-10$ & $2.21 \mathrm{e}-10$ \\
\hline 6 & 1.89 & .5 & 179.55 & $1.29 \mathrm{e}-04$ & $5.10 \mathrm{e}-05$ \\
\hline 7 & .0001 & NA & 227.22 & $1.01 \mathrm{e}-03$ & $4.25 \mathrm{e}-04$ \\
\hline 7 & .9 & NA & 288.03 & $2.85 \mathrm{e}-03$ & $6.43 \mathrm{e}-03$ \\
\hline 7 & .99999 & NA & 399.82 & $1.54 \mathrm{e}-02$ & $6.43 \mathrm{e}-03$ \\
\hline 8 &. .0896 & 0. & 54.97 & $8.31 \mathrm{e}-10$ & $4.03 \mathrm{e}-10$ \\
\hline U & NA & NA & 227.22 & $1.01 \mathrm{e}-03$ & $4.25 \mathrm{e}-04$ \\
\hline
\end{tabular}

Table 4: $y=\exp \left(\sigma^{2}\left(x-x_{0}\right)^{2} / 2\right), x_{0}=.9$

\begin{tabular}{||c|c|c|c|c|c||}
\hline Mapping & $\alpha_{1}$ & $\alpha_{2}$ & $I_{2}$ & Max Error & $L_{2}$ Error \\
\hline 6 & 5.136 & .0738 & 386.52 & $2.75 \mathrm{e}-03$ & $1.37 \mathrm{e}-03$ \\
\hline 7 & .99999 & NA & 567.2 & $1.56 \mathrm{e}-02$ & $8.17 \mathrm{e}-03$ \\
\hline 7 & .90000 & NA & 787.1 & $7.81 \mathrm{e}-02$ & $2.60 \mathrm{e}-02$ \\
\hline U & NA & NA & 1031.0 & $1.70 \mathrm{e}-01$ & $5.54 \mathrm{e}-02$ \\
\hline
\end{tabular}

Table 5: Sum of two Gaussians, $x_{0}=.30, x_{1}=-.15$

(7) are insensitive to $\alpha_{1}$.

The results using (8) were found to be very insensitive to $\alpha_{2}$ and are presented only for the mapping with $\alpha_{2}=0$ as it used in [5] for functions with rapid variation near the boundary. The results indicate that this mapping is effective in approximating boundary layer type solutions however it does not seem to offer any advantages over (6) and is not considered further.

We next consider the following function

$$
\left.\left.y=\exp \left(\sigma^{2}\left(x-x_{0}\right)\right)^{2} / 2\right)+\exp \left(\sigma^{2}\left(x-x_{1}\right)\right)^{2} / 2\right)
$$

The objective will be to determine the behavior of the various mappings when there is more than one region of rapid variation. The separation of the two regions of rapid variation can be controlled by varying $x_{0}$ and $x_{1}$. We first consider the case $x_{0}=.30, x_{1}=-.15, \sigma=50$. The results are presented in Table 5 .

It can be seen from the table that the family of mappings described by (6) yields a more accurate Chebyshev approximation even in the case of relatively separated pulses. We note that the minimum error of the mapping (7) in this case again appears to occur when $\alpha_{1} \rightarrow 1$ so that the collocation points are nearly uniformly spaced but the mapping is almost singular. We have also exhibited the degradation in accuracy obtained by reducing $\alpha_{1}$ from 1 by specifying an upper limit on $\alpha_{1}$ of 0.9 . Even with this degradation the results obtained from using (7) are considerably better than for the case when no mapping is employed. 


\begin{tabular}{||c|c|c|c|c|c||}
\hline Mapping & $\alpha_{1}$ & $\alpha_{2}$ & $I_{2}$ & Max Error & $L_{2}$ Error \\
\hline 6 & 2.704 & .0780 & 625.4 & $4.73 \mathrm{e}-02$ & $1.43 \mathrm{e}-02$ \\
\hline 7 & .99999 & NA & 567.1 & $1.45 \mathrm{e}-02$ & $7.96 \mathrm{e}-03$ \\
\hline 7 & .90000 & NA & 758.8 & $5.76 \mathrm{e}-02$ & $2.35 \mathrm{e}-02$ \\
\hline U & NA & NA & 1018.04 & $1.95 \mathrm{e}-01$ & $4.84 \mathrm{e}-02$ \\
\hline
\end{tabular}

Table 6: Sum of two Gaussians, $x_{0}=.50, x_{1}=-.25$

\begin{tabular}{||c|c|c|c|c|c||}
\hline Mapping & $\alpha_{1}$ & $\alpha_{2}$ & $I_{2}$ & Max Error & $L_{2}$ Error \\
\hline 6 & 3.172 & .361 & 375.3 & $3.86 \mathrm{e}-03$ & $1.57 \mathrm{e}-03$ \\
\hline 7 & .99999 & NA & 566.6 & $1.43 \mathrm{e}-02$ & $7.71 \mathrm{e}-03$ \\
\hline 7 & .90000 & NA & 629.3 & $8.01 \mathrm{e}-02$ & $1.99 \mathrm{e}-02$ \\
\hline U & NA & NA & 742.91 & $1.44 \mathrm{e}-01$ & $3.78 \mathrm{e}-02$ \\
\hline
\end{tabular}

Table 7: Sum of two Gaussians, $x_{0}=.90, x_{1}=.2$

We next consider more widely separated pulses by setting $x_{0}=.5, x_{1}=-.25, \sigma=$ 40. The results are presented in Table 6. This is a relatively severe case as neither of the pulses are located close to the boundary. We observe that for widely separated pulses located away from the boundaries the use of (6) leads to larger errors than (7), provided values of $\alpha_{1}$ near 1 are used. The results obtained from (7) are not strongly sensitive to $x_{0}$. This is not surprising as the effect of the mapping is to make the collocation points more uniform (in $x$ ).

We finally consider the case where one of the pulses is located near the boundary. In this case we consider $x_{0}=.9, x_{1}=.2, \sigma=50$. The results are presented in Table 7. We note that the best approximation occurs when $\alpha_{2}$ is close to the location of the interior spike.

In the case of multiple regions of rapid variation the optimum values of $\alpha_{1}$ and $\alpha_{2}$ for the mapping (6) are not suggested by the locations of the regions of rapid variation. These parameters must be determined by a numerical minimization procedure.

In summary our results indicate that the strategy of transforming the given function to one that is more readily approximated by a polynomial, which led to the derivation of the family of mappings (6) appears to be more effective than the strategy of mapping points of rapid variation to the boundaries. In particular the use of (6) appears to be superior in all respects to (8). The parameter $\alpha_{2}$ in (6) is an excellent indicator of the location of maximum variation, but the mapping is very robust if $\alpha_{2}$ is varied away from this location.

When the rapid variation occurs away from the boundary the use of (7) with $\alpha_{1}$ near 1 so that the collocation points are nearly uniformly spaced is preferable to the 


\begin{tabular}{||c|c|c|c|c|c||}
\hline Mapping & $\alpha_{1}$ & $\alpha_{2}$ & $I_{2}$ & Max Error & $L_{2}$ Error \\
\hline 6 & 6.54 & 0. & 3.61 & $6.00 \mathrm{e}-9$ & $4.23 \mathrm{e}-9$ \\
\hline 7 & .99624 & NA & 7.99 & $3.66 \mathrm{e}-05$ & $3.06 \mathrm{e}-04$ \\
\hline U & NA & NA & 14.59 & $7.80 \mathrm{e}-04$ & $5.36 \mathrm{e}-04$ \\
\hline
\end{tabular}

Table 8: $y=0.05 /\left(x^{2}+0.05\right)$

unmapped Chebyshev method. In these cases the mapping (7) is most accurate as $\alpha_{1} \rightarrow 1$ and degrades when $\alpha_{1}$ is reduced from the singular value. It is therefore preferable to use this mapping as close to the singular point as possible. When there is a single region of rapid variation near the boundaries the unmapped Chebyshev approximation is more accurate than the use of (7).

The use of (6) is superior to (7) for functions with rapid variation except when there are multiple regions of rapid variations which are widely separated and not located near the boundaries. In this case the best strategy seems to be to use (7) to obtain nearly equally spaced collocation points. Although this analysis is presented for pulse like functions similar effects hold for other types of functions with regions of rapid variation, for example functions which exhibit a step function type of behavior or localized highly oscillatory behavior.

In this paper we have concentrated on the role of mappings in enhancing the accuracy of Chebyshev pseudo-spectral approximations. The transformation (7) was originally introduced to decrease the spectral radius of the spectral differentiation operator and thereby increase the allowable timestep in the solution of time dependent partial differential equations.

Although the major emphasis in this paper is on the approximation of rapidly varying functions we have found that the mapping (6) is also effective in approximating functions which vary more gradually. We illustrate this with the example

$$
y=0.05 /\left(x^{2}+0.05\right),
$$

which was also considered in [13]. In this case we use 32 collocation points. The results of the approximation are shown in Table 8.

We next illustrate the effectiveness of this mapping in approximating solutions of partial differential equations. We consider the Burgers equation

$$
u_{t}+u u_{x}=\nu u_{x x},
$$

where $-1 \leq x \leq 1$. The initial condition and the boundary conditions are chosen as

$$
u(x, 0)=-\sin \pi x, \quad u( \pm 1, t)=0 .
$$

The viscosity coefficient $\nu=.01 / \pi$. This problem has been used as test case for a variety of spectral, pseudo-spectral and finite difference methods $[2,9]$. As $t$ increases 


\begin{tabular}{||c|c|c||}
\hline$m$ & $\pi t$ & $\alpha_{1}$ \\
\hline 151.9896 & 1.60407 & 21.5 \\
\hline 152.3214 & 1.60404 & 37.0 \\
\hline
\end{tabular}

Table 9: Burgers equation with $\mathrm{N}=61$

the solution develops a very steep gradient at the $x=0$. The maximum gradient at $x=0$ occurs when $\pi t$ is close to 1.6. As $t$ increases beyond this point the solution relaxes to zero. We have solved this problem using an adaptive pseudo-spectral method together with the mapping (6). The solution is symmetric about $x=0$ and the minimum of (3) always occurs at $\alpha_{2}=0$.

The solution to this problem can be computed analytically. One measure of accuracy, which has been used in $[2,9]$ is the quantity

$$
m=\max \left(u_{x}(0, t)\right) \text {. }
$$

The analytic result quoted in [2] is $m=1.5200516$ occuring at $\pi t=1.6037$. We have found analogously to what was found in [9] that the coordinate system which yields the most accurate calculation of $m$ is not perfectly predicted by the adaptive procedure. Minimizing the functional (3) appears to concentrate too much resolution near $x=0$ at the expense of resolution away from $x=0$. A similar conclusion was arrived at in [9] using an adaptive procedure based on minimizing a different functional. When the search region is artificially constricted so that the parameter $\alpha_{1}$ does not get too large, effectively reducing the resolution near $x=0$. we find excellent agreement with the analytic value for $m$. We indicate the computed values of $m$ in Table 9. The number of collocation points, $N$, was 61 . The first entry in the table is the result when the value of $\alpha_{1}$ was restricted while the second entry is for the value of $\alpha_{1}$ selected by the adaptive procedure.

Using the mapping (6) a high degree of accuracy can be attained as the number of collocation points is reduced. We indicate this in table (10) where we list the values of $m$ obtained for different numbers of collocation points. In this table we have not restricted the search for $\alpha_{1}$ but rather taken the value of $\alpha_{1}$ predicted by minimizing (3). In most calculations the exact solution is not available and the minimization of some functional is at present the only practical method to predict the appropriate coordinate system.

\section{Multiple Domains}

The results presented above indicate that the mapping (6) can significantly improve the Chebyshev approximation to rapidly varying function when there is only one region of rapid variation or when there are several closely spaced regions of rapid variation. When there are widely spaced regions of rapid variation away from the boundaries the effectiveness of (6) degrades and the best strategy within the con- 


\begin{tabular}{||c|c|c|c||}
\hline$m$ & $\pi t$ & $\alpha_{1}$ & $\mathrm{~N}$ \\
\hline 151.7508 & 1.61622 & 37.0 & 33 \\
\hline 152.3011 & 1.60446 & 37.0 & 49 \\
\hline 152.3214 & 1.60404 & 37.0 & 61 \\
\hline
\end{tabular}

Table 10: Burgers equation varying $\mathrm{N}$

text of a single domain is to use (7) with $\alpha$ near 1 so as to obtain a nearly uniform distribution of collocation points. However the accuracy obtained from using (7) is generally less than the accuracy that could be obtained from use of the mapping (6) if the regions of rapid variation were not widely separated.

One possible approach to this problem is to extend the mapping (6) to allow for the resolution of multiple regions of rapid variation. Such an extension would require additional parameters in the mapping and therefore the resulting minimization problem to determine appropriate parameters would be considerably more expensive than for the case when the mapping depends on only two parameters.

An alternative approach is to introduce two or more subdomains and employ mappings within each subdomain. The use of multiple domains can lead to an improvement in accuracy by (a) resolving small scale structures in the problem by introducing domains corresponding to the length scales appropriate to the problem, (b) choosing the interface so that the small scale structures or rapid variations occur near the boundary, and (c) isolating different regions of rapid variations within each subdomain and then employing mappings such as (6) within each subdomain in order to improve the accuracy of the unmapped Chebyshev approximation.

Multiple domain procedures have other advantages in addition to possible improvements in accuracy, for example they lend themselves to parallel computation and can lead to smaller and better conditioned matrices, see for example $[8,15,12]$. In this paper however, we will only consider the effect of multiple domains on the accuracy of approximation of rapidly varying functions. In particular we will demonstrate that a strategy such as that described by point (c) above, involving the interaction of mappings and domain decomposition can lead to significant improvements in the accuracy of Chebyshev interpolation.

In the context of the solution of partial differential equations, the use of multiple domains introduces additional parameters which have to be determined, in particular the number of domains, the location of the interface points, and the number of collocation points within each subdomain. If the multiple domains are chosen to correspond to very small scale structures or localized rapid variations, then the accuracy of the resulting approximation may be may be quite sensitive to the location and extent of the interface points. These parameters are generally chosen non-adaptively or with prior knowledge of the behavior of the function to be approximated.

When mappings are employed additional parameters must be determined. For example, if there are two domains and the mapping (6) is employed in each sub- 
domain then there are 7 parameters which have to be determined (the parameters of the mapping, the location of the interface and the number of collocation points within each subdomain). In this paper we will constrain the number of points so that each domain has an equal number of points. This strategy is preferable for parallel computation, although it may not necessarily be preferable for determining the most accurate approximation with a minimum number of computational degrees of freedom. We will also constrain the number of domains to be 2 . We now require the determination of 5 parameters, together with some procedure to determine the location of the interface, e.g. some functional which measures the error as a function of the location of the interface. The determination of these parameters could be significantly simplified if the location of the domain could be determined independently of the mapping parameters within each subdomain, i.e. a strategy whereby the location of the domain is determined from the current solution, and then new mapping parameters are determined within each subdomain by the minimization procedure described above. We will demonstrate that a strategy of employing multiple domains to isolate regions of rapid variation, together with the mapping (6) to resolve localized regions of rapid variation within each subdomain can lead to such a decoupling of the problem of determining these parameters.

In order to accomplish this, it is first necessary to understand the role of the location of the interface on the accuracy of the approximation and to develop functionals which can monitor this. We will therefore first consider the use of multiple domains without any mapping within each subdomain and then demonstrate that once an appropriate interface is found the errors in the approximation can be further reduced by employing mappings such as (6) within each subdomain. We will consider the approximation of (11) with $\sigma=40$ and with 41 points within each subdomain. We will further restrict ourselves to the case where $x_{0}=.5, x_{1}=-.25$ although similar results have been obtained for other values of these parameters. The accuracy of single domain Chebyshev approximations to this function under various mappings is described above. We use 41 points within each subdomain and compute the largest of the maximum norm errors.

The location of the interface, $Q$, will be obtained adaptively by determining $Q$ so that the maximum of (3) in each subdomain is minimized. Since we use an equal number of points in each subdomain, this corresponds to minimizing an estimate for the largest of the maximum norm errors. In Table 11 we show that this strategy leads to an improvement in accuracy over the single domain result and in the case where the interface is chosen in an ad hoc manner to be equidistant from both spikes, i. e. $Q=\left(x_{0}+x_{1}\right) / 2$. We have then employed the mapping (6) within each subdomain to determine parameters $\alpha_{1}^{1}, \alpha_{2}^{1}, \alpha_{1}^{2}, \alpha_{2}^{2}$, determined to minimize (3) in each subdomain as in Section 4 above (we employ a simple linear mapping to map each subdomain to the interval $I$ ).

It is apparent from the Table 11 that the overall accuracy of the approximation is very sensitive to the location of the interface when no mapping is employed. In this case the strategy of minimizing the maximum, over the two domains, of (3) 


\begin{tabular}{||c|c|c|c|c|c|c|c|c||}
\hline$I_{2}^{1}$ & $I_{2}^{2}$ & $Q$ & Mapping & $\alpha_{1}^{1}$ & $\alpha_{2}^{1}$ & $\alpha_{1}^{2}$ & $\alpha_{2}^{2}$ & Max Error \\
\hline 1018.04 & \multicolumn{2}{|c|}{ single domain } & U & NA & NA & NA & NA & $1.95 \mathrm{e}-01$ \\
\hline 625.4 & \multicolumn{2}{|c|}{ single domain } & $(6)$ & 2.7404 & .0780 & NA & NA & $4.73 \mathrm{e}-02$ \\
\hline 567.1 & \multicolumn{2}{|c|}{ single domain } & $(7)$ & .99999 & NA & NA & NA & $7.96 \mathrm{e}-03$ \\
\hline 215.8 & 159.1 & .125 & U & NA & NA & NA & NA & $1.08 \mathrm{e}-01$ \\
\hline 17.52 & 17.24 & .125 & $(6)$ & 20.60 & .330 & 15.70 & -.1417 & $5.17 \mathrm{e}-06$ \\
\hline 183.0 & 181.8 & .05 & U & NA & NA & NA & NA & $6.44 \mathrm{e}-02$ \\
\hline 17.25 & 17.56 & .05 & $(6)$ & 47.75 & .425 & 17.47 & -.0473 & $4.59 \mathrm{e}-06$ \\
\hline 150.27 & 131.05 & .05 & $(7)$ & .99999 & NA & .99999 & NA & $3.29 \mathrm{e}-02$ \\
\hline
\end{tabular}

Table 11: Effect of mappings on multi-domain approximation

leads to a significant reduction of the error.

The use of the mapping (6) can lead to a dramatic reduction in the error when there are multiple domains. In this case the overall accuracy of the Chebyshev interpolation is not sensitive to the location of the interface as long as the domains are such that there are not widely separated regions of rapid variation within each domain. In the solution of partial differential equations it may be important to determine the domains adaptively so that the regions of rapid variation are localized within each subdomain. The strategy of minimizing the maximum of (3) appears to accomplish this. Although we illustrate this behavior for only one example we have observed this behavior for other cases, not reported here, as well.

The use of (7) leads to an improvement in accuracy over the unmapped Chebyshev approximation but the improvement is not as great as in the case of the single domain. We note that the results obtained using (7) are slightly worse than in the unmapped case. This is due to the fact that the collocation points are nearly uniformly spaced and since $Q \neq 0$, the spacing of the collocation points is greater in one of the subdomains. Setting $Q=0$ we get errors very similar to the single domain case.

In summary, results indicate that the use of the mapping (6) in conjunction with a multiple domain procedure can lead to a improvement in Chebyshev interpolation and pseudo-spectral methods for the case that the function to be approximated has multiple regions of rapid variation. Furthermore, this improvement can be obtained from adaptive procedures without prior knowledge of the location of the rapid variation.

The mapping (6) can be extended to higher dimensions to deal with highly curved regions of rapid variation. One way this can be done is by allowing $\alpha$ to depend on the transverse coordinates similar to the procedure employed in [4]. Domain decomposition in several dimensions is usually based on rectangular domains. In this case such regions will not always be contained in one subdomain. 


\section{References}

[1] J. Augenbaum, Appl. Num. Math. $\underline{5}, 459$ (1989).

[2] C. Basdevant et. al., Computers and Fluids 14, 23 (1986).

[3] A. Bayliss, D. Gottlieb, B. J. Matkowsky, and M. Minkoff, J. Comput. Phys. 81, 421 (1989).

[4] A. Bayliss, R. Kuske, and B. J. Matkowsky, J. Comput. Phys., to appear.

[5] A. Bayliss and B. J. Matkowsky, J. Comput. Phys. 71, 147. (1987).

[6] J.M. Burger, The Nonlinear Diffusion Equation (Reidel Publ. Co., Boston, 1975).

[7] W. Cai, D. Gottlieb and C. Shu, Math. Comp. 므, 389 (1989).

[8] C. Canuto, M. Y. Husaini, A Quarteroni and T. A. Zang, Spectral Methods in Fluid Dynamics (Springer-Verlag, New York, 1987).

[9] H. Guillard and R. Peyret, Comput. Methods Appl. Mech. Engrg. 66, 17 (1988).

[10] D. Gottlieb, in Proc. $9^{\text {th }}$ Int. Conf. Numerical Methods in Fluid Dynamics edited by Soubbarameyer and Boujot (Springer, New York, 1985), p.48.

[11] D. Gottlieb and S. A. Orszag, Numerical Analysis of Spectral Methods: Theory and Applications (C.B.M.S. - N.S.F. Conference Series in Applied Mathematics, SIAM, Philadelphia, 1977).

[12] D. A. Kopriva, SIAM J. Sci. Stat. Comput. 10, 120 (1989).

[13] D. Kosloff and H. Tal-Ezer, ICASE Report No. 89-71 (1989).

[14] J. H. McCabe and G. M. Phillips, BIT $\underline{13}, 434$ (1973).

[15] M. G. Macareg and C. L. Streett, Appl. Num. Math. 2 95 (1986).

[16] A. Solomonoff and E. Turkel, J. Comput. Phys. $\underline{81,} 239$ (1989). 


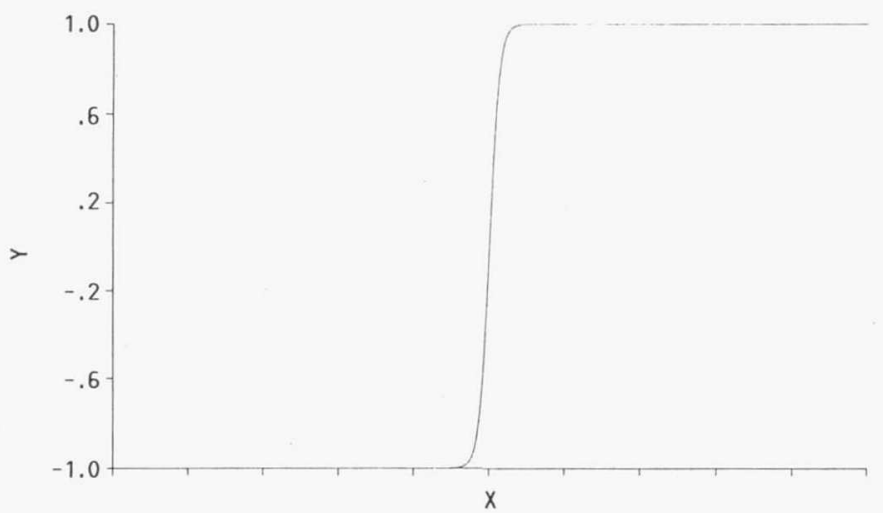

FIGURE 1a. (9) PLOTTED AGAINST THE INDEPENDENT VARIABLE $X$.

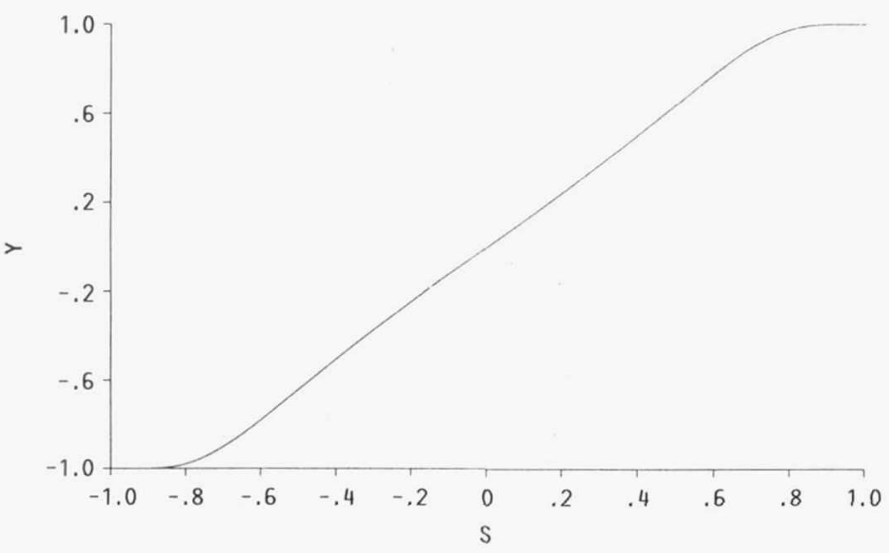

FIGURE 1b. (9) PLOTTED AGAINST THE TRANSFORMED VARIABLE S.

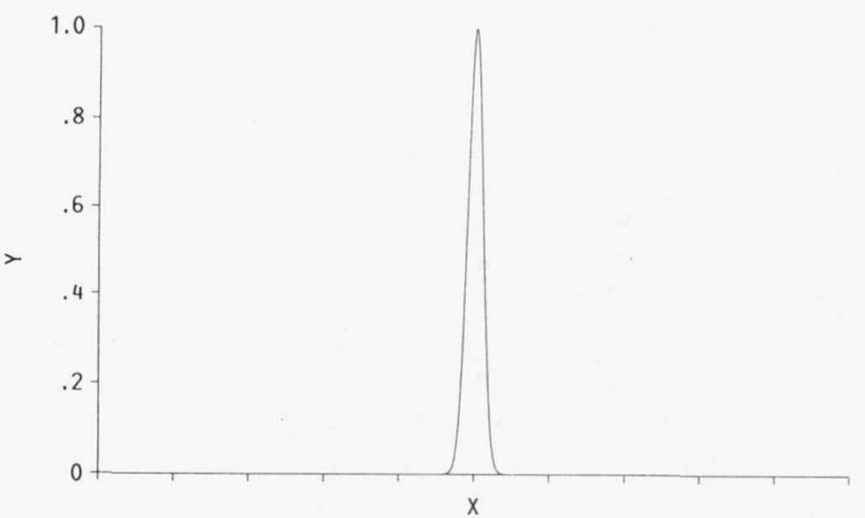

FIgURE 2a. (10) PLOTTED AGAINST THE INDEPENDENT VARIABLE $X$.

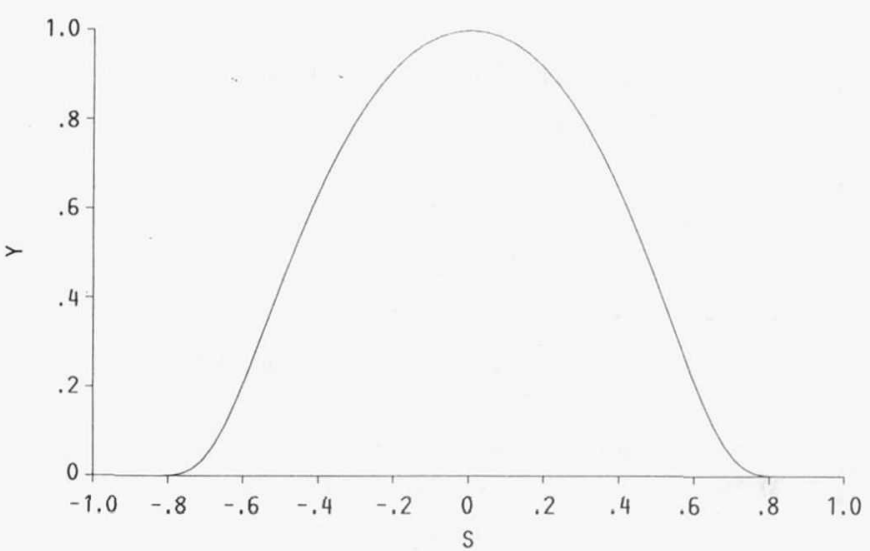

FIGURE 2b. (10) PLOTTED AGAINST THE TRANSFORMED VARIABLE S. 


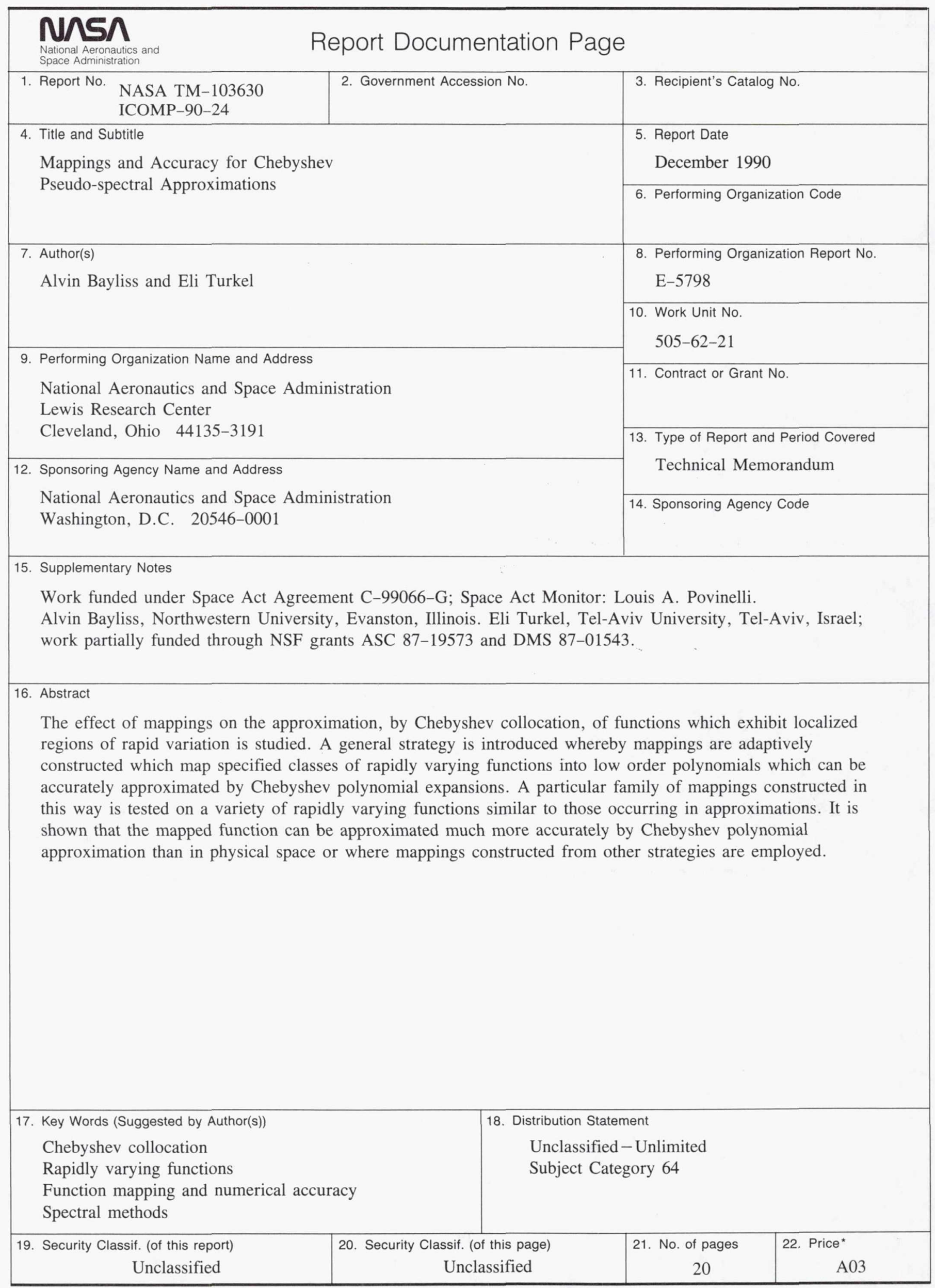

